\title{
Sleep-Wake Differences in Heart Rate Variability During a 105-Day Simulated Mission to Mars
}

\author{
Daniel E. Vigo, Barbara Ogrinz, Li Wan, Evgeny Bersenev, \\ Francis Tuerlinckx, OMer VAN DEN Bergh, \\ And André E. Aubert
}

\begin{abstract}
Vigo De, Ogrinz B, Wan L, Bersenev E, Tuerlinckx F, Van den BERGH O, AUBERT AE. Sleep-wake differences in heart rate variability during a 105-day simulated mission to Mars. Aviat Space Environ Med 2012; 83:125-30.

Introduction: In prolonged spaceflights the effect of long-term confinement on the autonomic regulation of the heart is difficult to separate from the effect of prolonged exposure to microgravity or other spacerelated stressors. Our objective was to investigate whether the sleepwake variations in the autonomic control of the heart are specifically altered by long-term confinement during the 105-d pilot study of the Earth-based Mars500 project. Methods: Before (pre), during (T1: 30, T2: 70, and T3: $100 \mathrm{~d}$ ), and after (post) confinement, 24-h EKG records were obtained from the six crewmembers that participated in the mission. Sleep and wake periods were determined by fitting a square wave to the data. Autonomic activity was evaluated through time and frequency domain indexes of heart rate variability (HRV) analysis during wake and sleep periods. Results: During confinement, wake HRV showed decreased mean heart rate and increased amplitude at all frequency levels, particularly in the very low (pre: $13.3 \pm 0.2 ; \mathrm{T} 1: 13.9 \pm 0.3 ; \mathrm{T} 2: 13.9 \pm$ $0.2 ; \mathrm{T} 3: 13.9 \pm 0.2 ;$ post: $13.2 \pm 0.2$ ) and high (pre: $7.6 \pm 0.4 ; \mathrm{T} 1: 8.3 \pm$ $0.5 ; \mathrm{T} 2: 8.2 \pm 0.4 ; \mathrm{T} 3: 8.1 \pm 0.4$; post: $7.6 \pm 0.3$ ) frequency components (values expressed as mean \pm SE of wavelet power coefficients). Sleep HRV remained constant, while sleep-wake high frequency HRV differences diminished. Discussion: The observed autonomic changes during confinement reflect an increase in parasympathetic activity during wake periods. Several factors could account for this observation, including reduced daylight exposure related to the confinement situation.

Keywords: autonomic nervous system, confinement, Mars500, space physiology.
\end{abstract}

GEVERAL ENVIRONMENTAL factors related to longSterm spaceflight, such as confinement, varying light exposure, noise or temperature changes, and absence of Earth-based Zeitgeber, are likely to play a role in sleep-wake cycle alterations (14). Some evidence has been found regarding the effect of confinement by itself on circadian rhythms. A 28-d isolation experiment revealed no major sleep disturbances besides an increase in selfrated tiredness (22). In contrast, during a 7- $d$ confinement period, catecholamines and sleep motor activity exhibited significant increases (13). Moreover, adaptation to changed physical and social environments during isolation periods of $135 \mathrm{~d}$ was associated with specific changes in sleep architecture (25).

Although autonomic nervous system (ANS) activity may play a key role in performance during space missions (3), little is known about the specific impact of confinement on the circadian rhythm of ANS activity. This rhythm is characterized by a sympathetic predominance during the wake periods that allows an active engagement with the external environment with increased usage of energy and a parasympathetic predominance during the night related to a disengagement from the external environment for recovery (18). A decrease in parasympathetic activity was reported in space (1) and ground (23) based bed-rest experiments with long periods of confinement. In an experiment conducted in order to characterize neurovegetative activity in a ground-based unit that simulated the living conditions of a space station except microgravity, no major differences were disclosed before, during, or after the isolation period of $60 \mathrm{~d}$ (16). Still, none of these studies evaluated the circadian rhythm of autonomic activity $(1,16,23)$.

Hence, we sought to investigate the circadian profile of heart rate variability during a 105-d confinement period in the context of the Mars500 pilot study (Mars105). The aim of the Mars500 project is to gather data, knowledge, and experience about the psychological and physiological effects of living in an Earthbased enclosed environment during the $520 \mathrm{~d}$ as would be required for a real mission to Mars. This allows separation of the effect of long-term confinement from the effect of long-term exposure to microgravity. We hypothesized that sleep-wake variations of autonomic control of the heart would be altered by long-term confinement.

From the Departamento de Docencia e Investigación, Facultad de Ciencias Médicas, Universidad Católica Argentina and Consejo Nacional de Investigaciones Científicas y Técnicas (CONICET), Buenos Aires, Argentina; the Department of Psychology, Katholieke Universiteit Leuven, Leuven, Belgium; the State Science Center of Russian Federation - Institute of Biomedical Problems of Russian Academia of Science, Moscow, Russia; and the Laboratory of Experimental Cardiology and Interdisciplinary Centre for Space Studies, Katholieke Universiteit Leuven, Leuven, Belgium.

This manuscript was received for review in June 2011. It was accepted for publication in November 2011.

Address correspondence and reprint requests to: André E. Aubert, M.D., Ph.D., Laboratory of Experimental Cardiology and Interdisciplinary Centre for Space Studies, Katholieke Universiteit Leuven, Herestraat 49, B-3000 Leuven, Belgium; andre.aubert@med.kuleuven.be and dvigo1973@gmail.com.

Reprint \& Copyright (C) by the Aerospace Medical Association, Alexandria, VA.

DOI: $10.3357 /$ ASEM.3120.2012 


\section{METHODS}

\section{Subjects}

Six healthy nonsmoking male subjects (mean \pm SD: age $33 \pm 6 \mathrm{yr}$; height $181 \pm 5 \mathrm{~cm}$; weight $82 \pm 12 \mathrm{~kg}$; BMI $25 \pm 3 \mathrm{~kg} \cdot \mathrm{m}^{-2}$ ) were selected to participate in a 105-d confinement pilot study before the Mars500 project.

\section{Design}

The Mars500 project, organized by the European Space Agency (ESA) and the Institute for Biomedical Problems (IBMP) at Moscow, is designed to simulate a mission to Mars in duration, composition of the crew, activities, workload, and communication facilities. The protocol of the study reported herein was approved in advance by the Ethics Committee of the University Hospital Gasthuisberg of Leuven, Belgium, and the ESA Medical Board, which complied with all guidelines stated in the Declaration of Helsinki. All participants gave informed consent to participate in the study.

Subjects were confined in the isolation facility at IBMP in Moscow from the $31^{\text {st }}$ of March 2009 to the $14^{\text {th }}$ of July 2009. The layout of the isolation facility comprises four hermetically sealed interconnected habitat modules with artificial lighting conditions (50-300 lx). The total volume of the habitat modules is $550 \mathrm{~m}^{3}$. Ambient temperature was maintained at a constant $24^{\circ} \mathrm{C}$, with a relative humidity of $35-45 \%$. Subjects were involved in different scientific protocols to assess the psychological and physiological effects of isolation and confinement. Their schedules were organized in order to maintain 8-h periods of work, leisure, and sleep. Crewmembers operated on night shifts for $1 \mathrm{wk}$ each, in rotation.

At five time points, 24-h Holter signals were obtained: in one day between 17 to $20 \mathrm{~d}$ before confinement (Pre); in one day between the $38^{\text {th }}$ to the $40^{\text {th }}$ day of confinement (T1); in one day between the $73^{\text {rd }}$ to the $76^{\text {th }}$ day of confinement (T2); in one day between the $98^{\text {th }}$ to the $100^{\text {th }}$ day of confinement (T3); and in one day between 11 to $13 \mathrm{~d}$ after the end of confinement (Post). Data collection was performed regardless of day or night shift of the subject.

\section{Procedure}

Signal recording: Electrocardiogram signal was recorded using a digital Holter device. Ventricular depolarizations ( $\mathrm{R}$ waves) were detected through the device software. The time elapsed between $R$ waves ( $R R$ intervals) was then computed. Heart rate variability (HRV) indexes were computed in 1-h segments. Premature and lost beats were identified by an automated filter and replaced by RR intervals resulting from linear interpolation (20).

Time domain: Quantitative time series analysis was performed on heart rate by evaluating measures of variation over time. Among these, $\mathrm{RR}_{\mathrm{m}}$ (mean duration of RR intervals in ms) quantifies the mean heart rate, SDNN (standard deviation of RR intervals in $\mathrm{ms}$ ) represents a coarse quantification of overall variability, and RMSSD (square root of the mean squared differences of successive normal RR) measures short-term heart rate variations (20).

Frequency domain: These measurements provide an evaluation of the power of the contributing frequencies underlying HRV. Its high-frequency (HF) component $(0.15-0.4 \mathrm{~Hz})$ is related to respiratory sinus arrhythmia and mediated by parasympathetic activity, whereas the low-frequency (LF) component $(0.04-0.15 \mathrm{~Hz})$ is related to baroreflex control and depends upon sympathetic and parasympathetic mechanisms. A very low frequency (VLF) component $(<0.04 \mathrm{~Hz})$ of an uncertain origin is also found and has been attributed to thermoregulatory fluctuations in vasomotor tone as well as to humoral factors such as the renin-angiotensin system, with dependence on the presence of parasympathetic outflow (Fig. 1) $(20,21)$.

To analyze the frequency components of HRV, the discrete wavelet transform (DWT) was chosen rather than the traditional fast Fourier transform because it is not affected by discontinuities or non-stationarities (2). Before applying the DWT, the linear trend and the mean value were subtracted from the signal. In addition, it was evenly sampled with a frequency of $2.4 \mathrm{~Hz}$ by means of a spline interpolation algorithm and zero padded to the next higher power of two (2). A six-level wavelet decomposition was employed to analyze the signal using a Daubechies four-wavelet function. Using this decomposition, wavelet levels A6 and D2-D6 represent the total power (TP, 0-0.6 Hz), wavelet levels A6 and D6 approximately correspond to the VLF band ( 0 $0.0375 \mathrm{~Hz})$, wavelet levels D4-D5 to the LF band (0.0375$0.15 \mathrm{~Hz})$, and wavelet levels D2-D3 to the HF band $(0.15-0.6 \mathrm{~Hz})$. In DWT, the square of the standard deviation of wavelet coefficients at each level is concordant with the spectral power of that level (2). Reported values are expressed as the natural logarithm of TP, HF, LF, and VLF; normalized units of LF [LF/ (TP - VLF) $\times 100$ ] and $\mathrm{HF}[\mathrm{HF} /(\mathrm{TP}-\mathrm{VLF}) \times 100]$; and the ratio between LF and HF. The use of normalized units minimizes the effect of the changes in TP on the values of LF and HF, and emphasizes the balanced behavior of the two branches of the autonomic nervous system (20).

Subjects' reports of waking and sleeping times and actigraphy records were not available for the pilot study. In general, visual inspection of the individual records showed a typical fall of heart rate during the night, with abrupt transitions between periods. These observations suggest a square wave model (two alternating contiguous periods of low and high heart rate) of the 24-h heart rate record. These variations are similar to those seen in 24-h beat-to-beat blood pressure recordings, where square wave modeling accounted for a larger fraction of circadian variance than modeling based on visual inspection, cosinor method, or fixed clock time (11).

Briefly, 20-min consecutive averages of RR-intervals were calculated. Square waves were constructed using all the possible different combinations of the low and high heart rate period lengths. Both the averaged signal and each square wave were standardized to a mean of 


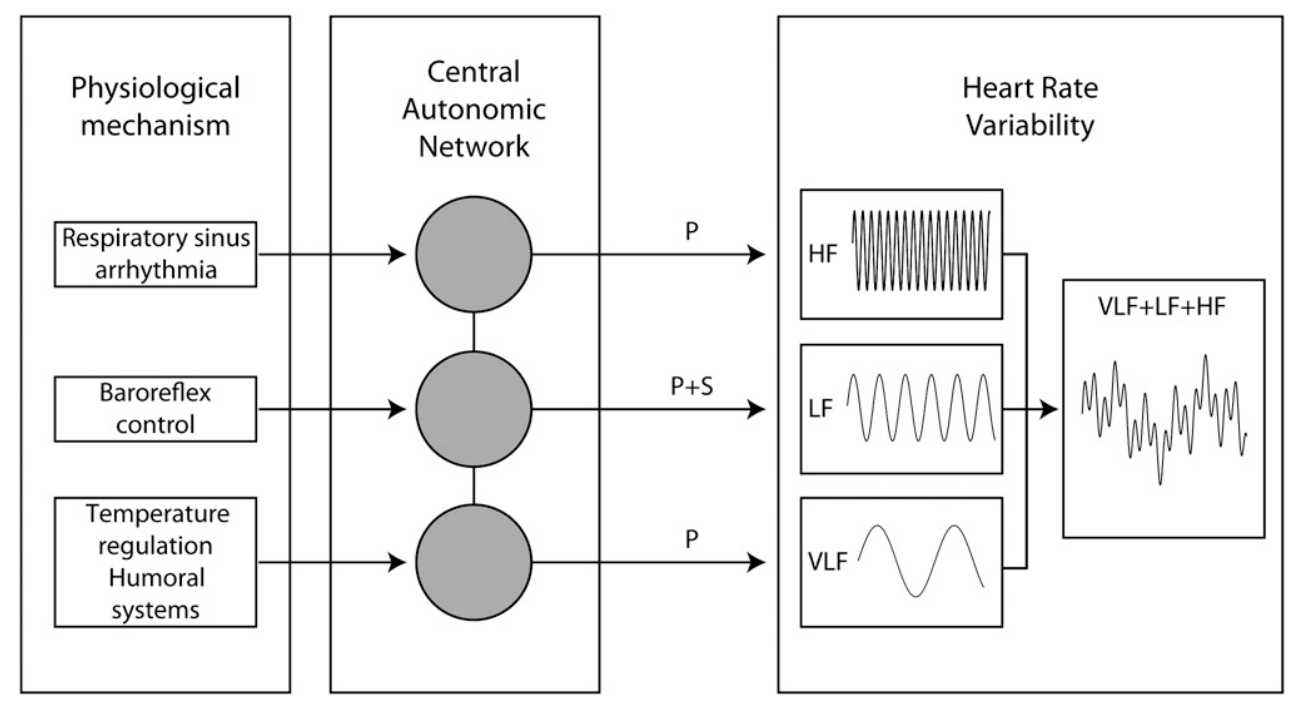

Fig. 1. Physiological mechanisms underlying heart rate variability. Respiratory rhythm is associated with high frequency changes of heart rate, mediated by parasympathetic activity; the baroreflex function is associated with low frequency changes of heart rate, mediated by sympathetic and parasympathetic activity; less settled processes like temperature regulation or endocrine systems are related with very low frequency changes of heart rate, with dependence on the presence of parasympathetic outflow. The analysis of the power of these rhythms permits inferences on the function of sympathetic and parasympathetic activity. Several structures are involved in the central autonomic control of these mechanisms, including the insular cortex, prefrontal cortex, hypothalamic areas, amygdala, midbrain and pons areas, and medullary nuclei. P: parasympathetic; S: sympathetic; VLF: very low frequency; LF: low frequency; HF high frequency.

zero and a standard deviation of 1.0. Cross correlation values of the standardized RR-interval signal with all possible different standardized square waves were determined. The best fitting square wave was identified by the highest cross-correlation value. This square wave was used to segment the original RR-interval record in a high (wake) and a low (sleep) heart rate period. The transience time from the high to the low heart rate period was identified as $t_{\text {down }}$ while the transience time from the low to the high heart rate period was identified as $t_{\text {up }}$ (Fig. 2) (11). The fit resulting from this model is optimal with respect to the square error. The square of the highest cross correlation value expresses the fraction of total variation of the 24-h RR-interval profile accounted for by the model (11).

In 4 of the 30 records a low heart rate period was identified by the model within the daytime (before 20:00). Visual inspection of these records revealed two different low heart rate periods better described by a biphasic square wave model. Therefore, the original record was split into a daytime record (before 20:00) and a nighttime record (after 20:00). The model was applied to these separate sections in order to finally construct a biphasic square wave with two different low (sleep) heart rate periods.

Hourly HRV was averaged along wake and nighttime sleep periods. HRV differences between night sleep and wake averages were also calculated. Each hour was assigned to a wake or to a sleep period according to the transience times previously defined. At least $55 \mathrm{~min}$ of any hour should fall within a specific period to be assigned to it; otherwise it was marked as a transition hour and excluded from the wake or sleep period average. Daytime sleep periods were marked as naps and excluded from the wake period average.

\section{Statistical Analysis}

Sleep-wake data and HRV indexes were expressed as mean \pm SE. Normality was assessed by means of a Kolmogorov-Smirnov test. A natural logarithm transform was used where needed. Initially, in order to assess the effect of confinement by itself, sleep-wake data, wake HRV, and sleep HRV indexes were averaged along non-confinement days (Pre and Post) and confinement days (T1, T2, T3). Differences between both conditions were evaluated through a paired-samples T-test. Then,

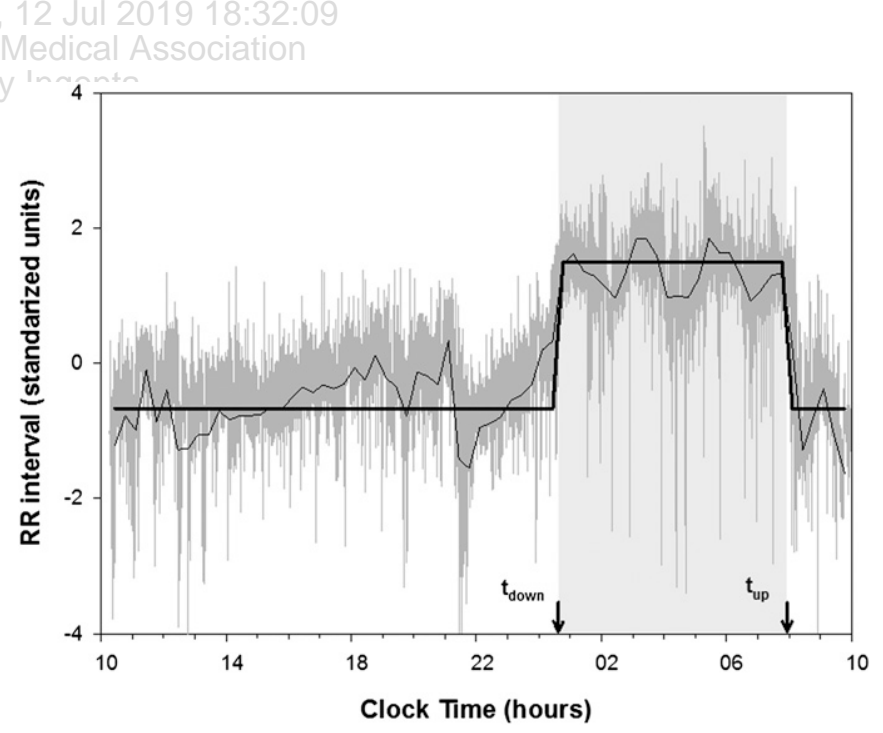

Fig. 2. Typical example of a 24-h recording of RR intervals standardized to a mean of zero and a standard deviation of 1 (dark grey line). The corresponding 20-min interval averaged signal (thin black line) and a square wave fit are also shown (thick black line). The transience time to the low and high heart rate periods are represented by $t_{\text {down }}$ and $t_{u p}$, as indicated by the square wave fit, with the sleep period (light gray area) within them. 
differences between measurement days (Pre, T1, T2, T3, and Post) were assessed by means of a repeated measures ANOVA test followed by a Tukey HSD post hoc test. A Mauchly's sphericity test was conducted in order to use a univariate approach for ANOVA analysis; when sphericity could not be assumed a multivariate approach was preferred.

\section{RESULTS}

The mean $t_{\text {down }}$ and $t_{\text {up }}$ time points varied non-significantly between midnight and 02:00 and between 06:00 and 09:00, respectively. These time points determined sleep periods with a mean duration of 6 to $8 \mathrm{~h}$. Only Subject 2 wore the Holter while he was awake on nightshift after 00:00, showing a short nighttime sleep period $3 \mathrm{~h}$ before midnight and no daytime sleep period. The fraction of the variance explained by the model varied non-significantly during isolation between 55\% and 75\% (not shown).

Confinement was associated with a diminished mean heart rate and an augmented global HRV during the day. While the increase in HRV was verified in all frequency components, the comparison between components revealed a relative decrease of LF. These changes seem to be more evident in the second month of isolation as revealed by the significant decrease in LF/HF in T2 (Table I). During sleep, RR interval duration showed an increase during confinement [Pre: $1088 \pm 62 \mathrm{~ms}$; T1: $1169 \pm 55$ ms; T2: $1211 \pm 60$ ms; T3: $1176 \pm 72$ ms; Post: $1112 \pm 58$ ms; F $(4,20)=3.45 ; P<0.05 ; \mathrm{T} 2$ different from Pre and Post]. However, no significant differences were found during measurement days in sleep HRV (not shown).

When comparing wake and sleep HRV, it was observed that confinement was associated with a reduction of HRV sleep-wake differences in SDNN, TP, VLF, LF, HF, and LF/HF. In addition, especially for TP and VLF, the sign of the mean difference changed (Table II). The analysis of contrasts between measurement days showed a significant decrease in RMSSD, HF, and LF/HF sleepwake differences. Post hoc pairwise comparisons between measurement days only revealed a significant reduction of HF-HRV sleep-wake difference, where T1 < Post, T2 $<$ Pre, and T2 $<$ Post (Table II).

\section{DISCUSSION}

The main result of the present study is that during confinement, wake HRV showed decreased mean heart rate and increased amplitude at all frequency levels, with a decrease in the normalized units of the LF HRV component. Sleep HRV remained constant. In addition, confinement was associated with a decrease of VLF HRV, LF HRV, HF HRV, and LF/HF sleep-wake differences, as well as with the appearance of negative TP HRV and VLF HRV sleep-wake differences. These changes seem to be more pronounced for HF HRV and in the middle of the confinement period. No significant differences were found in the length or phase of the sleep-wake periods.

Heart rate oscillations at all frequency levels (VLF, LF, and HF) reflect parasympathetic influences $(20,21)$, while LF fluctuations are also tightly coupled with synchronous oscillations of efferent sympathetic nervous activity (21). Thus, the increase in HRV at all frequency bands, with a relative decrease in the LF component during confinement, can be explained by augmented parasympathetic activity with a loss of sympathetic predominance during wake periods. The increased vagal predominance during the day can also account for the vanishing of the sleep-wake differences of SDNN, LF and HF (usually positive), and LF/HF (usually negative), as well as for the appearance of negative sleep wake differences of TP and VLF (usually positive) (10).

These results are in line with observations reported in space analogue environments like Antarctica. In a 40-d stay in the Italian Antarctic Station of Terra Nova Bay, a relatively significant decrease of LF was found, which was interpreted as reduced sympathetic activity. This was associated with a significant reduction of the anterior pituitary and adrenal hormonal levels of the pituitary-adrenal hormonal axis. In addition, only daytime HRV values were different from baseline measurements, while nighttime measurements only differed within the isolation period (7).

Several factors may account for the observed results. Operational demands or social and recreational activities could cause sleep disturbances in spaceflights (5)

TABLE I. WAKE HRV.

\begin{tabular}{|c|c|c|c|c|c|c|c|}
\hline & Pre & T1 & T2 & T3 & Post & Conf. T (5) & Day $F(4,20)$ \\
\hline $\mathrm{RR}_{\mathrm{m}}(\mathrm{ms})$ & $780 \pm 37$ & $908 \pm 36$ & $944 \pm 46^{\S, \uparrow}$ & $896 \pm 54$ & $797 \pm 57$ & -2.42 & $4.73^{+}$ \\
\hline SDNN (ms) & $92 \pm 10$ & $128 \pm 17 \S$ & $120 \pm 13 \S$ & $121 \pm 15^{\S, q}$ & $90 \pm 10$ & $-3.78 *$ & $7.68^{+}$ \\
\hline $\operatorname{RMSSD}(\mathrm{ms})$ & $31 \pm 5$ & $46 \pm 9$ & $46 \pm 8$ & $42 \pm 9$ & $31 \pm 5$ & -2.39 & $4.55^{+}$ \\
\hline In TP (wpc) & $13.4 \pm 0.2$ & $14.0 \pm 0.3^{\S, \Phi}$ & $13.9 \pm 0.2^{\S, \boldsymbol{\top}}$ & $13.9 \pm 0.2^{\S, \uparrow}$ & $13.3 \pm 0.2$ & $-4.21^{+}$ & $9.31^{\ddagger}$ \\
\hline In VLF (wpc) & $13.3 \pm 0.2$ & $13.9 \pm 0.3_{\S}^{\S}$ & $13.9 \pm 0.2 \S$, & $13.9 \pm 0.2 \S$ ฯ & $13.2 \pm 0.2$ & $-4.18^{\dagger}$ & $9.18^{\ddagger}$ \\
\hline In LF (wpc) & $10.4 \pm 0.2$ & $10.9 \pm 0.3^{\S, \Phi}$ & $10.7 \pm 0.2$ & $10.7 \pm 0.3$ & $10.3 \pm 0.2$ & $-3.79 *$ & $5.51^{\dagger}$ \\
\hline In HF (wpc) & $7.6 \pm 0.4$ & $8.3 \pm 0.5 \S$ & $8.2 \pm 0.4 \S$ ฯ & $8.1 \pm 0.4$ & $7.6 \pm 0.3$ & $-4.09^{+}$ & $8.52^{\ddagger}$ \\
\hline LF (nu) & $93.5 \pm 1.0$ & $92.2 \pm 1.3$ & $92.0 \pm 1.2 \S$ & $92.7 \pm 1.1$ & $93.3 \pm 0.7$ & $2.80 *$ & $3.90 *$ \\
\hline $\mathrm{HF}(\mathrm{nu})$ & $6.5 \pm 1.0$ & $7.8 \pm 1.3$ & $8.0 \pm 1.2^{\S}$ & $7.3 \pm 1.1$ & $6.7 \pm 0.7$ & $-2.80 *$ & $3.90 *$ \\
\hline LF/HF & $17.1 \pm 3.0$ & $14.9 \pm 3.0$ & $13.9 \pm 2.2^{\S, \boldsymbol{\top}}$ & $15.4 \pm 2.5$ & $16.5 \pm 2.6$ & $3.00 *$ & $5.03^{\dagger}$ \\
\hline
\end{tabular}

Values are expressed as mean \pm SE. Conf.: contrast between confinement and non-confinement (paired-samples $t$-test); Day: contrast between measurement days (repeated measures ANOVA followed by Tukey HSD post hoc test); $\mathrm{RR}_{\mathrm{m}}$, mean of RR interval duration; SDNN, standard deviation of RR intervals; RMSSD, square root of the mean squared differences of successive normal RR; TP, total area power; VLF, very low frequency power; LF, low frequency power; HF, high frequency power; wpc, wavelet power coefficients; nu, normalized units. ${ }^{*} P<0.05 ;+P<0.01 ; \neq P<0.001 ; \S$ different from Pre; ${ }^{2}$ different from Post. 
TABLE II. SLEEP-WAKE HRV DIFFERENCES.

\begin{tabular}{|c|c|c|c|c|c|c|c|}
\hline & Pre & T1 & T2 & T3 & Post & Conf. T (5) & Day $F(4,20)$ \\
\hline $\mathrm{RR}_{\mathrm{m}}(\mathrm{ms})$ & $308 \pm 40$ & $261 \pm 30$ & $267 \pm 36$ & $281 \pm 25$ & $315 \pm 20$ & 1.72 & 1.15 \\
\hline SDNN (ms) & $16 \pm 9$ & $5 \pm 7$ & $-4 \pm 9$ & $-8 \pm 6$ & $23 \pm 10$ & $4.30^{+}$ & 2.47 \\
\hline RMSSD (ms) & $23 \pm 7$ & $11 \pm 2$ & $8 \pm 5$ & $13 \pm 3$ & $25 \pm 6$ & 2.38 & $2.96 *$ \\
\hline In TP (wpc) & $0.1 \pm 0.2$ & $-0.1 \pm 0.1$ & $-0.3 \pm 0.1$ & $-0.3 \pm 0.1$ & $0.2 \pm 0.2$ & $3.76 *$ & 2.34 \\
\hline In VLF (wpc) & $0.1 \pm 0.2$ & $-0.1 \pm 0.1$ & $-0.3 \pm 0.1$ & $-0.3 \pm 0.1$ & $0.2 \pm 0.2$ & $3.56 *$ & 2.20 \\
\hline In LF (wpc) & $0.4 \pm 0.2$ & $0.2 \pm 0.1$ & $0.1 \pm 0.1$ & $0.2 \pm 0.2$ & $0.5 \pm 0.1$ & $3.48 *$ & 1.74 \\
\hline In HF (wpc) & $0.8 \pm 0.2$ & $0.2 \pm 0.1 \S$ & $0.1 \pm 0.2^{\ddagger, \S}$ & $0.3 \pm 0.1$ & $0.9 \pm 0.1$ & $3.67 *$ & $5.70^{+}$ \\
\hline LF (nu) & $-1.3 \pm 2.0$ & $-1.3 \pm 0.7$ & $-1.8 \pm 1.1$ & $-2.5 \pm 1.5$ & $-2.0 \pm 1.6$ & 2.06 & 0.12 \\
\hline $\mathrm{HF}(\mathrm{nu})$ & $1.3 \pm 2.0$ & $1.3 \pm 0.7$ & $1.8 \pm 1.1$ & $2.5 \pm 1.5$ & $2.0 \pm 1.6$ & -2.06 & 0.12 \\
\hline $\mathrm{LF} / \mathrm{HF}$ & $-4.2 \pm 0.7$ & $0.5 \pm 1.4$ & $1.0 \pm 1.6$ & $-1.1 \pm 1.7$ & $-4.0 \pm 2.8$ & $-4.45^{\dagger}$ & $3.91 *$ \\
\hline
\end{tabular}

Values are expressed as mean \pm SE. Conf.: contrast between confinement and non-confinement (paired-samples t-test); Day: contrast between measurement days (repeated measures ANOVA followed by Tukey HSD post hoc test); $R_{\mathrm{m}}$, mean of RR interval duration; SDNN, standard deviation of RR intervals; RMSSD, square root of the mean squared differences of successive normal RR; TP, total area power; VLF, very low frequency power; LF, low frequency power; HF, high frequency power; wpc, wavelet power coefficients; nu, normalized units. ${ }^{*} P<0.05 ;+P<0.01$; $\neq$ different from pre; $\S$ different from post.

that in turn may disrupt other circadian physiological rhythms (14). However, poor sleep quality is associated with daytime reductions in HF HRV and heartbeat intervals (12) that contrast with the present observations. Moreover, we failed to demonstrate significant differences in sleep period length and phase.

Varying light exposure and reduced sensitivity to Zeitgeber strength during space missions were also associated with circadian rhythm disruptions. It is known that light is the dominant environmental input affecting circadian rhythms (14). Sympathetic activity during the day increases with light color temperature (26) and light intensity (27). In this regard, the loss of sympathetic predominance reported herein may be associated with a prolonged exposure to the artificial environmental light of the isolation facility, of lower intensity and color temperature than natural light.

Changes in mood related to confinement stressors like loneliness, boredom, or social stress should also be considered factors that may explain the observed results. During a winter in Antarctica it was reported that tension, anxiety, depression, anger, and confusion decline during the first half of the isolation period and increase close to the end of isolation (17). Psychophysiological data from this pilot study presented by other authors showed that, although mood tends to decrease until day 77 of isolation, differences within the $105 \mathrm{~d}$ of confinement were not significant (19). Also, apart from mean heart rate, no changes were seen in sleep HRV indexes, which are known to be sensitive markers to psychological stressors (9). Thus, the effect of mood changes in the observed results, if any, seems to be small.

Physical training is another factor that may be associated with changes in HRV. Confinement of almost any kind is associated with decreased physical load, even with implementation of special exercise programs. However, opposite to what is seen in the present study, HF HRV is reduced in sedentary subjects when compared to physically active ones (8).

The differences between confinement and non-confinement days could be magnified by a combined effect of increasing tension in anticipation of being locked up
(Pre) plus increased exposure to environmental and social demands after the confinement (Post). In this regard, the reduced environmental stimulation and regularly paced, quite predictable, and well-structured activity schedules during the confinement could be interpreted as a less stressful and healthier situation (4). However, increased vagal tone by itself should not necessarily be considered an index of increased health, since it can also be seen in pathologic conditions such as panic disorder (15).

Although Holter measurements were performed almost exclusively on day shifts, the effect of the night shifts in ANS activity should be taken into consideration. When compared with day work, shift work is associated with an increase in sympathetic activity either during wake periods (increase in heart rate and LF\%, decrease in SDNN) or during sleep periods (decrease in SDNN) (24). Therefore, the increase of parasympathetic activity associated with long-term confinement reported herein is unlikely to be due to the rotating shift work regime. The 520-d study will provide more data to analyze if the present results are modified by taking into account the daytime sleep periods associated with night shifts.

The results from the present study may be important since performance is associated with autonomic arousal. Specifically, it has been reported that the increase in LF HRV and HF HRV (as seen during confinement) is related to decreased attentional processing evaluated through an attentional load test (d2 test) (6). Objective physiological measures could be used to characterize differences in operational efficiency and in abilities to adapt to extreme environments. In turn, fatigue-related performance decrements caused by sleep loss or sustained operations might be improved with training to regulate crewmember responses, including autonomic and central nervous system parameters (3).

Several limitations should be considered. First, conclusions are restricted due to the small numbers of subjects, typical of this kind of research. Also, it remains to be seen how these results would translate to real space missions, where there is always the possibility of not 
getting back to Earth, an important stressor by itself. In addition, the precise determination of sleep-wake periods requires other techniques such as the recording of actigraphic data, not accessible due to the limitations of experimental procedures within this pilot study. Finally, the observed pattern of autonomic activity could be part of an infradian rhythm that could only be evidenced in longer confinement periods.

To conclude, data obtained during the 105-d isolation experiment revealed a loss of sympathetic predominance during wake periods. Several factors could account for this observation, including reduced daylight exposure related to the confinement situation. Further studies should address if decreased autonomic arousal may affect cognitive performance during long-term space missions.

\section{ACKNOWLEDGMENTS}

We wish to thank Marcelo de Nóbrega and Luis Issolio (Instituto de Investigación en Luz Ambiente y Visión - UNT-CONICET) for their advice about artificial lighting conditions. This work was supported by grants from the Belgian Federal Office of Scientitic Affairs through an ESA-PRODEX grant and from the Agencia Nacional de Promoción Científica y Tecnológica, Argentina (ANPCyT) (PICT 2007-01045). Daniel E. Vigo is a Research Career Awardee from the Argentine Research Council (CONICET). Li Wan is supported as a post-doctoral researcher through an ESA-PRODEX grant from the Belgian Federal Office of Scientific Affairs.

Authors and affiliations: Daniel E. Vigo, M.D., Ph.D., Departamento de Docencia e Investigación, Facultad de Ciencias Médicas, Universidad Católica Argentina - Consejo Nacional de Investigaciones Científicas y Técnicas (CONICET), Buenos Aires, Argentina; Barbara Ogrinz, M.A., Li Wan, M.D., Ph.D., Francis Tuerlinckx, Ph.D., Omer Van den Bergh, Ph.D., Department of Psychology, and André E. Aubert, M.D. Ph.D., Laboratory of Experimental Cardiology and Interdisciplinary Centre for Space Studies, Katholieke Universiteit Leuven, Leuven, Belgium; and Evgeny Bersenev, Ph.D., State Science Center of Russian Federation - Institute of Biomedical Problems of Russian Academia of Sciences, Moscow, Russia.

\section{REFERENCES}

1. Baevsky RM, Baranov VM, Funtova II, Diedrich A, Pashenko AV, et al. Autonomic cardiovascular and respiratory control during prolonged spaceflights aboard the International Space Station. J Appl Physiol 2007; 103:156-61.

2. Chan HL, Huang HH, Lin JL. Time-Frequency analysis of heart rate variability during transient segments. Ann Biomed Eng 2001; 29:983-96.

3. Cowings P, Toscano W, Deroshia C, Taylor B, Hines A, et al. Converging indicators for assessing individual differences in adaptation to extreme environments. Aviat Space Environ Med 2007; 78(5, Suppl.)B195-215.

4. de Gouw HW, Westendorp RG, Kunst AE, Mackenbach JP, Vandenbroucke JP. Decreased mortality among contemplative monks in The Netherlands. Am J Epidemiol 1995; 141:771-5.

5. Dijk DJ, Neri DF, Wyatt JK, Ronda JM, Riel E, et al. Sleep, performance, circadian rhythms, and light-dark cycles during two space shuttle flights. Am J Physiol Regul Integr Comp Physiol 2001; 281:R1647-64.

6. Duschek S, Muckenthaler M, Werner N, Del Paso GA. Relationships between features of autonomic cardiovascular control and cognitive performance. Biol Psychol 2009; 81:110-7.

7. Farrace S, Ferrara M, De AC, Trezza R, Cenni P, et al. Reduced sympathetic outflow and adrenal secretory activity during a 40-day stay in the Antarctic. Int J Psychophysiol 2003; 49:17-27.
8. Goldsmith RL, Bigger JT Jr, Steinman RC, Fleiss JL. Comparison of 24-hour parasympathetic activity in endurance-trained and untrained young men. J Am Coll Cardiol 1992; 20: 552-8.

9. Hall M, Vasko R, Buysse D, Ombao H, Chen Q, et al. Acute stress affects heart rate variability during sleep. Psychosom Med 2004; 66:56-62.

10. Huikuri HV, Niemela MJ, Ojala S, Rantala A, Ikaheimo MJ, Airaksinen KE. Circadian rhythms of frequency domain measures of heart rate variability in healthy subjects and patients with coronary artery disease. Effects of arousal and upright posture. Circulation 1994; 90:121-6.

11. Idema RN, Gelsema ES, Wenting GJ, Grashuis JL, van den Meiracker AH, et al. A new model for diurnal blood pressure profiling. Square wave fit compared with conventional methods. Hypertension 1992; 19(6, Pt. 1):595-605.

12. Kageyama T, Nishikido N, Kobayashi T, Kurokawa Y, Kaneko T, Kabuto M. Self-reported sleep quality, job stress, and daytime autonomic activities assessed in terms of short-term heart rate variability among male white-collar workers. Ind Health 1998; 36:263-72.

13. Kraft NO, Inoue N, Mizuno K, Ohshima H, Murai T, Sekiguchi C. Physiological changes, sleep, and morning mood in an isolated environment. Aviat Space Environ Med 2002; 73:1089-93.

14. Mallis MM, DeRoshia CW. Circadian rhythms, sleep, and performance in space. Aviat Space Environ Med 2005; 76(6, Suppl.)B94-107.

15. McCraty R, Atkinson M, Tomasino D, Stuppy WP. Analysis of twenty-four hour heart rate variability in patients with panic disorder. Biol Psychol 2001; 56:131-50.

16. Pagani M, Iellamo F, Lucini D, Pizzinelli P, Castrucci F, et al. Adaptational changes in the neural control of cardiorespiratory function in a confined environment: the CNEC\#3 experiment. Acta Astronaut 1995; 36:449-61.

17. Palinkas LA, Houseal M. Stages of change in mood and behavior during a winter in Antarctica. Environ Behav 2000; 32:12841.

18. Recordati G. A thermodynamic model of the sympathetic and parasympathetic nervous systems. Auton Neurosci 2003; 103:1-12.

19. Schneider S, Brummer V, Carnahan H, Kleinert J, Piacentini MF, et al. Exercise as a countermeasure to psycho-physiological deconditioning during long-term confinement. Behav Brain Res 2010; 211:208-14.

20. Seely AJ, Macklem PT. Complex systems and the technology of variability analysis. Crit Care 2004; 8:R367-84.

21. Taylor JA, Carr DL, Myers CW, Eckberg DL. Mechanisms underlying very-low-frequency RR-interval oscillations in humans. Circulation 1998; 98:547-55.

22. Tobler I, Borbely AA. European isolation and confinement study. Twenty-four hour rhythm of rest/activity and sleep/ wakefulness: comparison of subjective and objective measures. Adv Space Biol Med 1993; 3:163-83.

23. Traon AP, Sigaudo D, Vasseur P, Maillet A, Fortrat JO, et al. Cardiovascular responses to orthostatic tests after a 42-day head-down bed-rest. Eur J Appl Physiol Occup Physiol 1998; 77:50-9.

24. van Amelsvoort LG, Schouten EG, Maan AC, Swenne CA, Kok FJ. Occupational determinants of heart rate variability. Int Arch Occup Environ Health 2000; 73:255-62.

25. Veĭn AM, Ponomarev IP, Eligulashvili TS, Levin I, Kovrov GV, Filimonov MI. [Features of the sleep/wakefulness cycle in long-term isolation]. Aviakosm Ekolog Med 1997; 31:36-41.

26. Yasukouchi A, Ishibashi K. Non-visual effects of the color temperature of fluorescent lamps on physiological aspects in humans. J Physiol Anthropol Appl Human Sci 2005; 24:41-3.

27. Yokoi M, Aoki K, Shimomura Y, Iwanaga K, Katsuura T. Exposure to bright light modifies HRV responses to mental tasks during nocturnal sleep deprivation. J Physiol Anthropol 2006; 25:153-61. 